\title{
Thermal Conductance of Pressed Contacts at Liquid Helium Temperatures
}

L. J. Salerno, P. Kittel and A. L. Spivak

May 1983

\section{LIBRARY COPY}

JUN $6 \quad 1983$

LANGLEY RESEARCH CENTER

LIBRARY, NASA

HAMPTON, VIRGINIA 


\section{Thermal Conductance of Pressed Contacts at Liquid Helium Temperatures}

L. J. Salerno,

P. Kittel, Ames Research Center, Moffett Field, California

A. L. Spivak, Trans-Bay Electronics, Richmond California 
THERMAL CONDUCTANCE OF PRESSED CONTACTS AT LIQUID HELIUM TEMPERATURES

L. J. Salerno* and P. Kitte1*

NASA Ames Research Center, Moffett Field, California

and

A. L. Spivak ${ }^{\dagger}$

Trans-Bay Electronics, Richmond, California

\section{Abstract}

The thermal contact conductance of a $0.4 \mu \mathrm{m}$ surface finish OFHC copper sample pair has been investigated from 1.6 to $3.8 \mathrm{~K}$ for a range of applied contact forces up to $670 \mathrm{~N}$. relation

Experimental data have been fitted to the

$$
\dot{Q}=\int \alpha T^{n} d T
$$

by assuming that the thermal contact conductance is a simple power function of the sample temperature.

It has been found that the conductance is proportional to $\mathrm{T}^{2}$ and that conductance increases with an increase in applied contact force. These results confirm earlier work.

\section{Introduction}

The optimum design of cryogenic instruments requires accurate thermal models. This is especially important for instruments where performance is sensitive to temperature. Infrared instruments such as the Infrared Astronomical Satellite (IRAS) and the Shuttle Infrared Telescope Facility (SIRTF) fall into this category. The present models are limited by a lack of knowledge of the low temperature thermal conductance of the bolted joints that are typically used in the instrumentto-system interface. Previous studies of pressed contacts though limited in scope have shown that the thermal conductance does not obey the Wiedemann-Franz law (that states that the ratio of thermal to electrical conductivities is proportional to the temperature). In this paper, an effort to characterize the thermal conductance of pressed contacts at liquid helium-4 temperatures is described. Specifically, the dependence of thernal contact conductance on applied force and temperature is discussed.

\section{Theory and Previous Work}

The phenomenon of thermal contact resistance is attributable to several factors; most notably, it is the consequence of contact being made only at discrete locations, rather than over the entire surface area. Ideally, the contact area is represented by the interface area of the surfaces themselves; however, a close examination reveals that

*Research Scientist.

tResearch Technician.

This paper is declared a work of the U.S. Government and therefore is in the public domain. even in the smoothest surfaces, irregularities exist which may restrict the contact area to as few as three discrete spots, irrespective of the sample dimensions. This theory is supported by experimental findings that contact resistance is dependent on the force of contact rather than contact area. ${ }^{1,2}$ As the contact force increases, the material deforms. Thus, the area of initial contact increases and new spots develop. The heat flow is constricted in the vicinity of the contact locations because of the narrowness of the effective areas of contact. This constriction is in large part responsible for the contact resistance. ${ }^{3,4}$ Estimates of the constriction resistance have been made for various assumed contact geometries by modeling the contacts as individual elements. ${ }^{5}$ By arranging the elements in groups of varying heights, the case of surface waviness can be accounted for as well. ${ }^{3}, 5$

A relation involving contact pressure and material hardness exists for determining the ratio of the surface area to the actual area of contact. However, the equivalent radius of the contact spot must be known and, at the present, there is no theoretical method available for general determination since each sample must be considered on an individual basis. ${ }^{4}$ The height of the contact gap is also significant and a method does exist for its estimation. ${ }^{3}$

Additionally, the presence of surface films or oxides contribute to the problem. This is especially significant in the case of materials such as aluminum, which form oxides immediately in the presence of air. In the case of oxides, the oxide layer must be penetrated to obtain a consistent measure of the thermal resistance.

Although there has been significant interest $^{3}, 5-10$ in the problem of contact resistance, estimation of the resistance from proposed theoretical models is still not a simple task. Principles of variational calculus have been applied to the problem to determine upper and lower bounds; however, because of the nature of the problem, there are limitations in the models and no satisfactory agreement exists between models due to the incompatibility of their boundary conditions. This poses a problem in predicting the behavior of pressed contacts and, therefore, most usable data in the field are empirical. Additionally, an attempt to predict thermal conductance from the electrical conductance given by the WiedemannFranz relation yields values much less than those obtained empirically. It has been found that this discrepancy can be as large as a factor of $5 \times 10^{6}$ for contacts made at $4.2 \mathrm{~K}$ (Ref. 1).

Previous work consists of limited data for pressed contacts in the $4 \mathrm{~K}$ region. ${ }^{1,2,11-14}$ of these data, $\mathrm{Cu}-\mathrm{Cu}$ results seem to dominate and, of the work surveyed, there seems to be good general 
agreement for the $\mathrm{Cu}-\mathrm{Cu}$ conductance under applied forces of $450 \mathrm{~N}$. Other work is limited to particular sample pairs and configurations, of ten corresponding to specific applications. ${ }^{9,15}$ A need exists for more general thermal conductance data covering a variety of samples over a range of temperatures, contact forces, and surface conditions.

\section{Experimental Procedure}

An apparatus has been fabricated and tested which has been used to measure the thermal conductance of pressed sample pairs at temperatures from $1.6 \mathrm{~K}$ to $4.2 \mathrm{~K}$ under applied forces of up to $700 \mathrm{~N}$. The apparatus is pictured in Figs. 1 and 2 . In operation, it is immersed in a Dewar filled with liquid helium 4 . To obtain data below $4.2 \mathrm{~K}$, the temperature of the liquid helium 4 is reduced by evaporative cooling. A pressure controller limits evacuation to achieve the desired temperature.

The following relation describes the mechanism of heat flow across the boundary between pressed solid surfaces:

$$
\dot{\mathrm{Q}}=\mathrm{k} \Delta \mathrm{T}
$$

where $\dot{Q}$ is the thermal energy transferred across the boundary, $k$ is the effective thermal conductance of the contact, and $\Delta \mathrm{T}$ is the temperature difference across the boundary. While Eq. (1) is valid at any particular temperature $T$, $k$ is actually a function of $T$ so that:

$$
\dot{Q}=\int k(T) d T
$$

If a simple power law is assumed to represent $k(T)$ and temperatures $\mathrm{Tu}$ and $\mathrm{T} \ell$ as measured at each side of the boundary, it follows that:

$$
\dot{Q}=\int_{T \ell}^{T u} \alpha T^{n} d T=\alpha\left(T u^{n+1}-T \ell^{n+1}\right) /(n+1)
$$

As discussed below $\alpha$ and $\mathrm{n}$ are determined empirically for each sample pair, using Eq. (3).

The actual pressed contact sample pairs were fabricated from OFHC copper. The samples were cleaned and stored in a nitrogen environment to prevent contamination while not under test. Five pairs were prepared to evaluate the effect of different surface finishes on thermal conductance. Surface finishes of $0.1,0.2,0.4,0.8$, and $1.6 \mu \mathrm{m}$ rms were selected. Each sample pair had the following dimensions: $10.2 \mathrm{~mm}$ diam and $10.2 \mathrm{~mm}$ length for the lower sample, and $12.7 \mathrm{~mm}$ diam and $8.89 \mathrm{~mm}$ length for the upper sample. The large diameter of the upper sample is to assure that any slight lateral movement would not prevent complete surface contact with the lower sample. Calibrated germanium resistance thermometers were installed in the upper and lower samples. A heater consisting of manganin wire wound on an aluminum form was placed above the upper sample (see Fig. 2). An analysis was performed to determine the losses of heater power due to radiation and gas conduction. This analysis showed such losses to be negligible, assuring that the measured heater power was the power actually applied. Each sample pair was tested from 1.6 to $4.2 \mathrm{~K}$ over the range from 0 to
$670 \mathrm{~N}$. For each temperature and force value, heater powers from $0.100 \mathrm{~mW}$ to $10.0 \mathrm{~mW}$ were applied, the upper and lower sample temperatures measured. The force was then automatically incremented to the next value. In this manner, data sets were obtained for both ascending and descending values of the applied force, to assist in determining the magnitude of a hysteresis effect (if any). It should be noted that the apparatus was in all cases cooled down from room temperature with essentially zero applied force. Loading of the sample was performed during data acquisition only, and the load was relaxed when changing bath temperatures.

\section{$\underline{\text { Results }}$}

Tables 1-8 present results obtained for the $0.4 \mu \mathrm{m}$ surface finish $\mathrm{Cu}-\mathrm{Cu}$ sample pair. The first column in the tables denotes the bath temperature at which the data were taken. (Tables 1-7 1ist results for both ascending and descending force values, while Table 8 gives the ascending value only, since $670 \mathrm{~N}$ is the upper force limit.) The next two columns give the derived values of $\alpha$ and $n$. These values were obtained from a computer program which fit the experimental values of heater power $\dot{Q}$, upper sample temperature $\mathrm{Tu}$, and lower sample temperature $\mathrm{Tl}$ to Eq. (3). The program also computes the magnitude of the offset in heater power Qo such that with no heater power applied a line fit of $\dot{Q}$ versus $\Delta T$ passes through the origin. The values of Qo are shown in column 3. In addition, the program performs a statistical analysis of the data in terms of the known uncertainties in the experimental measurements to calculate an uncertainty in the computed quantities $\alpha, n$, and $\dot{Q} \circ$. The next three columns give the standard deviation in the computed quantities $\alpha, n$, and $Q \circ$ as a measure of this error. The last two columns represent the minimum and maximum temperatures over which the computed values are accurate, representing the temperature range over which the original data were taken.

In addition to the values of $\alpha, n$, and $\dot{Q} \circ$, Tables $1-8$ provide the respective error associated with these values. By specifying the uncertainty in the experimental data due to measurement accuracy and round-off errors, and perturbing these data assuming a Gaussian distribution, a standard deviation of the values' input to the computer program is obtained. Employing a random number generator, 99 computations of $\alpha, n$, and $\dot{Q}_{\circ}$ were performed by the program within the standard deviation in the output values of $\alpha, n$, and $Q$. Fig. 3 the effective thermal conductance is plotted as a function of temperature for a range of applied forces. The curves shown in Fig. 3 were generated by averaging the values of $\alpha$ and $n$ for both ascending and descending values of a particular force and plotting $\alpha \mathrm{T}^{\mathrm{n}}$ as a function of temperature.

\section{Discussion}

The values of the exponent $n$ given in Tables 1-8 correspond well with earlier work. Berman $^{1}$ observed a nearly $\mathrm{T}^{2}$ temperature dependence of thermal conductance at liquid helium temperatures. The present range of values $\mathrm{n}=1.9$ to 2.2 certainly supports this finding. 
Comparing the results in Fig. 3 with those of Berman $^{1}$ shows that in particular, at an applied force of $670 \mathrm{~N}(150 \mathrm{lb})$, the value of $8.0 \times 10^{-3} \mathrm{~W} / \mathrm{K}$ is within a factor of 2 of Berman's value $1.46 \times 10^{-2} \mathrm{~W} / \mathrm{K}$. Since Berman simply specifies a machine finish, it is felt that satisfactory agreement exists. Also shown is the effect of increasing applied force on thermal conductance. It is evident that the thermal conductance very definitely increases with increasing force, again supporting the earlier work of Berman.

\section{Conclusions}

It has been shown that for the $0.4 \mu \mathrm{m}$ OFHC copper pressed contact pair, the thermal conductance varies roughly as the second power of the temperature, and increases with increasing applied force.

Future work will focus on copper sample pairs of differing finishes as well as stainless steel, aluminum, and brass and silica glass samples. Also, the effect of such coatings as gold and indium on thermal contact conductance w11l be investigated.

\section{Acknowledgments}

The authors wish to acknowledge the contributions of C. Banda, G. Villere, and especially W. G. Marks, of Informatics, Inc., for their assistance in the area of software development.

\section{$\underline{\text { References }}$}

${ }^{1}$ Berman, R., "Some Experiments on Thermal Contact at Low Temperatures," J. App1. Physics, Vol. 27, No. 4, 1956.

${ }^{2}$ Berman, R. and Mate, C. F., "Thermal Contact at Low Temperatures," Nature, Dec. 13, 1958.

${ }^{3}$ Bobeth, M. and Diener, G., "Variational Bounds for the Effective Thermal Contact Resistance Between Bodies with Rough Surfaces," Int. J. Heat Mass Trnsf., Vo1. 25, No. 1, 1982, pp. 111-117.

${ }^{4}$ Jeng, D. R., "Thermal Contact Resistance in Vacuum," J. Heat Transfer, Trans. ASME, Aug. 1967, pp. $275-276$.

Table 1 Copper sample pair $0.4 \mu \mathrm{m}$ surface finish $22 \mathrm{~N}$ applied force

\begin{tabular}{|c|c|c|c|c|c|c|c|c|}
\hline 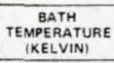 & $\alpha\left(m w / x^{n+1}\right)$ & ${ }^{\circ}$ & $a_{0}(\mathrm{mw})$ & $s a$ & s. & s. $0_{0}$ & 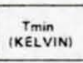 & $\begin{array}{c}\operatorname{Tmax} \\
\text { (KELLVIN) }\end{array}$ \\
\hline $\begin{array}{c}3.8 \\
\text { SECENG } \\
\text { OESCENONG } \\
\end{array}$ & $\begin{array}{l}0.1600 \\
0.1649 \\
\end{array}$ & $\begin{array}{l}1.911 \\
1.917 \\
\end{array}$ & $\begin{array}{rrr}6.663 & E-2 \\
7.678 & E-2 \\
\end{array}$ & $\begin{array}{rl}7.309 & E .3 \\
1.967 & E .3 \\
\end{array}$ & $\begin{array}{rl}1,902 & E .3 \\
7.486 & E_{3}\end{array}$ & $\begin{aligned} & 1,208 \begin{array}{c}E-3 \\
7.487 \\
E-3\end{array} \\
&\end{aligned}$ & $\begin{array}{l}3.781 \\
3.781\end{array}$ & $\begin{array}{l}5.014 \\
5.982 \\
\end{array}$ \\
\hline $\begin{array}{c}3.6 \\
\text { ASCENDING } \\
\text { DESCENDING } \\
\end{array}$ & $\begin{array}{l}0.1552 \\
0.1784 \\
\end{array}$ & $\begin{array}{l}1.928 \\
1.882 \\
\end{array}$ & $\begin{array}{l}7.144 \\
1.371 \\
\varepsilon, 2.2 \\
\end{array}$ & 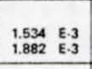 & $\begin{array}{ll}6.290 & E .3 \\
6.742 & E .3\end{array}$ & $\begin{array}{rll}1.107 & E .3 \\
1.274 & E \cdot 3\end{array}$ & $\begin{array}{l}3.576 \\
3.576 \\
\end{array}$ & $\begin{array}{r}5.968 \\
5.968 \\
\end{array}$ \\
\hline $\begin{array}{c}3.4 \\
\text { ASCENDING } \\
\text { OESCENOING }\end{array}$ & \begin{tabular}{|l}
0.1516 \\
0.1742 \\
\end{tabular} & $\begin{array}{l}1.946 \\
1.893 \\
\end{array}$ & $\begin{array}{lll}8.329 & E .2 \\
1.568 & E-1\end{array}$ & $\begin{array}{l}1.312 \quad E-3 \\
1.594 \quad E=3\end{array}$ & $\begin{array}{rl}5.507 & E .3 \\
5.937 & E .3 \\
\end{array}$ & $\begin{array}{ccc}1.052 & E-3 \\
1.221 & E-3\end{array}$ & $\begin{array}{l}3.376 \\
3.379\end{array}$ & $\begin{array}{l}5.891 \\
5.824\end{array}$ \\
\hline $\begin{array}{c}3.2 \\
\text { ASEENDING } \\
\text { OESCENDING }\end{array}$ & $\begin{array}{l}0.1504 \\
0.1704 \\
\end{array}$ & $\begin{array}{l}1.948 \\
1.901 \\
\end{array}$ & $\begin{array}{lll}8.911 & \varepsilon \cdot 2 \\
1.552 & E-1 \\
\end{array}$ & 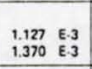 & 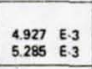 & $\begin{array}{rl}9.936 & E .4 \\
1.144 & E .3 \\
\end{array}$ & $\begin{array}{l}3.178 \\
3.211\end{array}$ & $\begin{array}{l}5.843 \\
5.7255 \\
\end{array}$ \\
\hline $\begin{array}{c}30 \\
\text { ASCENDING } \\
\text { DESCENOING }\end{array}$ & $\begin{array}{r}0.1434 \\
0.1595 \\
\end{array}$ & $\begin{array}{l}1.991 \\
1.932 \\
\end{array}$ & $\begin{array}{rl}8.5455 & E \cdot 2 \\
1.439 & E-1 \\
\end{array}$ & $\begin{array}{lll}9.5282 & E-4 \\
1.118 & E .3 \\
\end{array}$ & $\begin{array}{rl}4.430 & E-3 \\
4.679 & E-3 \\
\end{array}$ & $\begin{array}{l}9.361 \quad E \cdot 4 \\
1.064 \\
E-3\end{array}$ & $\begin{array}{l}2.987 \\
3.002 \\
\end{array}$ & $\begin{array}{r}5.810 \\
5.749 \\
\end{array}$ \\
\hline $\begin{array}{l}2.4 \\
\text { ASCENDING } \\
\text { DESCENDING }\end{array}$ & $=$ & $=$ & $=$ & : & $=$ & $=$ & = & $=$ \\
\hline $\begin{array}{l}20 \\
\text { ASCENDING } \\
\text { OESCENDING }\end{array}$ & : & $=$ & E & : & 三 & - & E & $=$ \\
\hline $\begin{array}{l}1.6 \\
\text { ASCENDING } \\
\text { OESCENDING }\end{array}$ & - & $=$ & $=$ & - & z & I & $\overline{-}$ & = \\
\hline
\end{tabular}

${ }^{5}$ Bobeth, M. and Diener, G., "Upper Bounds for the Effective Thermal Contact Resistance Between Bodies with Rough Surfaces," Int. J. Heat Mass Trnsf., Vo1. 25, No. 8, 1982, pp. 1231-1238.

${ }^{6}$ Cooper, M. G., Mikiv, B. B., and Yovanovich, M. M., "Thermal Contact Conductance," Int. J. Heat Mass Trnsf., Vol. 12, 1969.

7Mian, M. N., Al-Astrabadi, F. R., O'Callaghan, P. W., and Probert, S. D., "Thermal Resistance of Pressed Contacts Between Steel Surfaces: Influence of Oxide Films," J. Mech. Eng. Sci., Vol. 21, No. 3, 1979.

${ }^{8}$ Mikesell, R. P. and Scott, R. B., "Heat Conduction Through Insulating Supports in Very Low Temperature Equipment," J. Research of Nat'1 Bureau Standards, Vol. 57, No. 6, Dec. 1956.

${ }^{9}$ Suomi, M., Anderson, A. C., and Holmstrom, B., "Heat Transfer Below $0.2 \mathrm{~K}$," Physica, Vol. 38, 1968.

${ }^{10}$ Thomas, T. R. and Probert, S. D., "Thermal Contact Resistance - the Directional Effect and Other Problems," Int. J. Heat Mass Trnsf., Vol. 13, 1970 .

${ }^{11}$ Deutsch, M., "Thermal Conductance in ScrewFastened Joints at Helium Temperatures," Cryogenics, May 1978.

${ }^{12}$ Manninen, M. and Zimmerman, W., "On the Use of Screw-Fastened Joints for Thermal Contact at Low Temperatures," Rev. Sci. Instr., Vo1. 48, No. $12,1977$.

${ }^{13}$ Radebaugh, R., Siegwarth, J. D., Lawless, W. N., and Morrow, A. J., "Electrocaloric Refrigeration for Superconductors," NSBIR 76-847, Feb. 1977.

${ }^{14}$ Wanner, M., "Thermal Conductance of a Pressed A1-A1 Contact," Cryogenics, Jan. 1981.

${ }^{15}$ Colwe11, J. H., "Thermal Contacts in a Low Temperature Cryostat," Cryogenics, Nov. 1973.

Table 2 Copper sample pair $0.4 \mu \mathrm{m}$ surface finish $45 \mathrm{~N}$ applied force

\begin{tabular}{|c|c|c|c|c|c|c|c|c|}
\hline $\begin{array}{l}\text { BATH } \\
\text { TEPERATRE } \\
\text { (KELVIN) }\end{array}$ & almiwikn & $n$ & $o_{0} \cdot m W \mid$ & s.o & s., & $5.0_{0}$ & 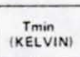 & Thax \\
\hline $\begin{array}{l}3.8 \\
\text { ASCENDING } \\
\text { OESCENDING }\end{array}$ & $\begin{array}{l}0.1722 \\
0.1847\end{array}$ & $\begin{array}{l}1.903 \\
1.911\end{array}$ & $\begin{array}{ll}7.004 & E-2 \\
7.576 & E .2\end{array}$ & $\begin{array}{l}2073 \quad E-3 \\
8.190 \quad E-3 \\
\end{array}$ & $\begin{array}{l}7.573 \\
8.190 \mathrm{E} \cdot-3\end{array}$ & $\begin{array}{l}1.243 \\
1.316 \\
\mathrm{E} .3\end{array}$ & $\begin{array}{l}3.780 \\
3.781\end{array}$ & $\begin{array}{l}5.952 \\
5.834 \\
\end{array}$ \\
\hline $\begin{array}{c}3.6 \\
\text { ASENDING } \\
\text { DESCENDING } \\
\end{array}$ & $\begin{array}{r}0.1632 \\
0.1986 \\
\end{array}$ & $\begin{array}{l}1.925 \\
1.879\end{array}$ & $\begin{array}{l}7.636 \\
1.351 \\
1.3 .1 \\
\end{array}$ & $\begin{array}{rl}1.671 & E-3 \\
2.250 & E-3 \\
\end{array}$ & $\begin{array}{rl}6.554 & E-3 \\
7.317 & E-3 \\
\end{array}$ & $\begin{array}{l}1.144 \\
1.335 \\
\mathrm{EE}_{3}\end{array}$ & $\begin{array}{l}3.576 \\
3.576\end{array}$ & $\begin{array}{l}5.898 \\
5.728\end{array}$ \\
\hline $\begin{array}{l}34 \\
\text { ASSENDING } \\
\text { DESCENDING }\end{array}$ & $\begin{array}{l}0.1631 \\
0.2001 \\
\end{array}$ & $\begin{array}{l}1.930 \\
1.871\end{array}$ & $\begin{array}{ll}1.019 & 5.1 \\
1.639 & E: 1\end{array}$ & $\begin{array}{ll}1.463 & E-3 \\
1.952 & E-3 \\
\end{array}$ & $\begin{array}{r}5.835 \\
6.404 \\
6.43 \\
E\end{array}$ & 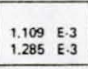 & $\begin{array}{l}3.375 \\
3.380 \\
3\end{array}$ & $\begin{array}{r}5819 \\
5.664 \\
\end{array}$ \\
\hline $\begin{array}{c}3.2 \\
\text { ASENOING } \\
\text { DESCENDING }\end{array}$ & $\begin{array}{l}0.1581 \\
0.1853 \\
\end{array}$ & $\begin{array}{l}1.948 \\
1.913 \\
\end{array}$ & $\begin{array}{l}1.083 \\
1.556 .1 \\
\text { E.1. }\end{array}$ & $\begin{array}{l}1.241 \\
1.576 \quad E .3 \\
\end{array}$ & $\begin{array}{ll}5.183 & E-3 \\
5.657 & E-3 \\
\end{array}$ & $\begin{array}{rl}1.052 & E .3 \\
1.192 & E .3 \\
\end{array}$ & $\begin{array}{l}3.18 \\
3.20 \\
\end{array}$ & $\begin{array}{l}5.768 \\
5.627 \\
\end{array}$ \\
\hline $\begin{array}{c}30 \\
\text { ASCENDING } \\
\text { DESCENDING } \\
\end{array}$ & $\begin{array}{l}0.1541 \\
0.1778 \\
\end{array}$ & $\begin{array}{l}1.959 \\
1.930\end{array}$ & $\begin{array}{rll}1.1113 & E .1 \\
1.468 & E-1 \\
\end{array}$ & $\begin{array}{rl}1.058 & E .3 \\
1.3000 & E=3 \\
\end{array}$ & $\begin{array}{l}4.603 \\
4.950 \\
\end{array}$ & $\begin{array}{rl}9.970 & E-4 \\
1.093 & E-3\end{array}$ & $\begin{array}{l}3.001 \\
3.001\end{array}$ & $\begin{array}{l}5.580 \\
5.580\end{array}$ \\
\hline $\begin{array}{c}2.4 \\
\text { ASEENDING } \\
\text { DESCENDING }\end{array}$ & 0.7464 E.1 & 1.889 & $4.711 \quad E \cdot 3$ & $3.881 \leq 4$ & $3.986 \mathrm{E} \cdot \mathrm{-3}$ & 2947 E.4 & 2572 & $\frac{4.802}{-}$ \\
\hline $\begin{array}{c}2.0 \\
\text { ASENDDNG } \\
\text { OESCENOING }\end{array}$ & Z & : & $\overline{-}$ & E & E & $=$ & $=$ & z \\
\hline $\begin{array}{c}1.6 \\
\text { ASCENDING } \\
\text { OESCENDING }\end{array}$ & - & $=$ & - & - & - & z & = & $=$ \\
\hline
\end{tabular}


Table 3 Copper sample pair $0.4 \mu \mathrm{m}$ surface finish $112 \mathrm{~N}$ applied force

\begin{tabular}{|c|c|c|c|c|c|c|c|c|}
\hline $\begin{array}{l}\text { BATH } \\
\text { TEMPEATURE } \\
\text { (KELVIN) }\end{array}$ & $\alpha\left(m w / K^{n+1}\right.$ & $n$ & $a_{0}(m w)$ & $s a$ & $\mathrm{sin}$ & $8 . a_{0}$ & $\begin{array}{c}\operatorname{T} \operatorname{Tin} \\
\text { (KELLVIN) }\end{array}$ & (KELVIN) \\
\hline \begin{tabular}{|c|c|}
3.8 \\
ASENDING \\
OESCENOING \\
\end{tabular} & $\begin{array}{l}0.2107 \\
0.2531\end{array}$ & \begin{tabular}{|l|l}
1.920 \\
1.891 \\
\end{tabular} & $\begin{array}{ll}6.521 & E-2 \\
7.156 & E-2 \\
\end{array}$ & \begin{tabular}{|l|l}
.095 & $E .3$ \\
1.076 & $E-2$ \\
\end{tabular} & 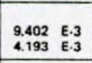 & 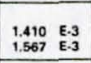 & $\begin{array}{l}3781 \\
3.780 \\
\end{array}$ & $\begin{array}{l}5.635 \\
5.464 \\
\end{array}$ \\
\hline $\begin{array}{c}3.6 \\
\text { ASCENDING } \\
\text { DESCENOING }\end{array}$ & 0.2043 & $\begin{array}{l}1.936 \\
1.920\end{array}$ & $\begin{array}{lll}8.173 & E-2 \\
1.261 & E-1\end{array}$ & $\begin{array}{ll}504 & E .3 \\
.523 & E .3\end{array}$ & $\begin{array}{l}8.021 \quad \mathrm{E} \cdot 3 \\
9.487 \\
\mathrm{E}, 3\end{array}$ & 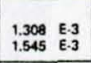 & $\begin{array}{l}3.576 \\
3.576 \\
\end{array}$ & $\begin{array}{l}7.477 \\
5.353\end{array}$ \\
\hline $\begin{array}{c}3.4 \\
\text { ASCENDING } \\
\text { DESCENDING }\end{array}$ & \begin{tabular}{|l|}
0.2026 \\
0.2459
\end{tabular} & \begin{tabular}{|l|l}
1.946 \\
1.921
\end{tabular} & $\begin{array}{lll}0.162 & E .1 \\
1.614 & E .1\end{array}$ & $\begin{array}{ll}2132 & E \cdot 3 \\
2.961 & E-3\end{array}$ & \begin{tabular}{|l|l|l}
7.023 & $E \cdot 3$ \\
8.142 & $E .3$
\end{tabular} & $\begin{array}{lll}1.265 & E-3 \\
1.473 & E-3\end{array}$ & $\begin{array}{l}3.377 \\
3.381\end{array}$ & $\begin{array}{l}5.471 \\
5.273\end{array}$ \\
\hline $\begin{array}{c}3.2 \\
\text { ASCENDING } \\
\text { DESCENDING }\end{array}$ & 0.2013 & 1.947 & 1.273 E.1 & 1.809 E.3 & $6.096 E-3$ & $1.191 \quad E-3$ & 3.185 & 5.416 \\
\hline \begin{tabular}{|c|}
30 \\
ASCENOING \\
DESCENOING
\end{tabular} & $\begin{array}{l}0.1984 \\
0.2246\end{array}$ & $\begin{array}{l}1.957 \\
1.969\end{array}$ & $\begin{array}{ll}1.281 & E .1 \\
1.447 & E-1\end{array}$ & $\begin{array}{l}1.5279, E \cdot 3 \\
1.924 \varepsilon^{z} \cdot 3\end{array}$ & $\begin{array}{lll}5.368 & E-3 \\
6.011 & E .3\end{array}$ & $\begin{array}{lll}1.1116 & E .3 \\
1.214 & E .3\end{array}$ & $\begin{array}{l}2989 \\
2.999\end{array}$ & $\begin{array}{l}5.364 \\
5.160\end{array}$ \\
\hline $\begin{array}{l}2.4 \\
\text { ASCENDING } \\
\text { DESCENOING }\end{array}$ & $\begin{array}{l}0.1135 \\
0.1479\end{array}$ & $\begin{array}{l}2020 \\
2060\end{array}$ & $\begin{array}{ll}-4.531 & E-2 \\
-7.869 & E-2\end{array}$ & $\begin{array}{ll}5.407 & E-4 \\
6.865 & E-4\end{array}$ & $\begin{array}{ll}3.254 & E \cdot 3 \\
3.175 & E-3\end{array}$ & $\begin{array}{ll}6.246 & E-4 \\
6.219 & E-4\end{array}$ & $\begin{array}{l}2.574 \\
2.584\end{array}$ & $\begin{array}{l}5.923 \\
5.896\end{array}$ \\
\hline $\begin{array}{c}2.0 \\
\text { ASCENOING } \\
\text { OESCENOING }\end{array}$ & - & = & E & $=$ & = & $=$ & = & = \\
\hline $\begin{array}{c}1.6 \\
\text { ASCENDING } \\
\text { OESCENDING }\end{array}$ & - & - & - & = & - & - & $\overline{-}$ & = \\
\hline
\end{tabular}

Table 4 Copper sample pair $0.4 \mu \mathrm{m}$ surface finish $224 \mathrm{~N}$ applied force

\begin{tabular}{|c|c|c|c|c|c|c|c|c|}
\hline $\begin{array}{r}\text { TEMPRE } \\
\text { KEELV }\end{array}$ & of & $n$ & $a_{0}(m \mathrm{~m})$ & 8.0 & s.n & $s, o_{0}$ & $\begin{array}{l}\text { Tinin } \\
\text { ELVIN) }\end{array}$ & $\begin{array}{l}T_{\max } \\
\text { ELVVIN }\end{array}$ \\
\hline & 0.2745 & $\begin{array}{l}1.967 \\
0.974\end{array}$ & $\begin{array}{ll}47 & E-2 \\
26 & E-2\end{array}$ & $\begin{array}{ll}5.626 & E_{3} \\
7.004 & E \cdot 3\end{array}$ & 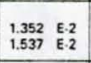 & $\begin{array}{l}7778-3 \\
.926 \\
E-3\end{array}$ & 3.7. & 5.239 \\
\hline & & & 1 & $3^{3}$ & \begin{tabular}{|l|l}
$E .2$ \\
$E$
\end{tabular} & & & $\begin{array}{l}5.139 \\
5.008\end{array}$ \\
\hline & .2696 & & $8 \varepsilon .1$ & 3 & $\begin{array}{rll}9.588 & E-3 \\
1.095 & E-2\end{array}$ & & & \\
\hline & 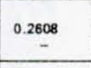 & 8 & - & & 8 & & & \\
\hline & & & & & & & & \\
\hline & & & & & & & & \\
\hline & & & & & & & & \\
\hline & & & $3 \in-2$ & 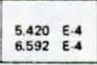 & $6 \mathrm{E} \cdot 3$ & 7.043 E.4 & 1.66 & $\begin{array}{l}5.506 \\
5.1999\end{array}$ \\
\hline
\end{tabular}

Table 5 Copper sample pair $0.4 \mu \mathrm{m}$ surface finish $336 \mathrm{~N}$ applied force

\begin{tabular}{|c|c|c|c|c|c|c|c|c|}
\hline $\begin{array}{l}\text { BATM } \\
\text { TEMERATURE } \\
\text { (KELVIN) }\end{array}$ & $\alpha\left(m w / k^{n+1}\right)$ & ${ }^{n}$ & imw & $s, \alpha$ & $1, n$ & $\$, 0_{0}$ & $\begin{array}{c}\text { TMmin } \\
\text { (KELVIN) }\end{array}$ & TELA \\
\hline & $\begin{array}{l}0.3173 \\
0.3135 \\
0\end{array}$ & 2008 & : & 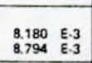 & $\begin{array}{rr}1.726 & \mathrm{E} \cdot 2 \\
1.838 & \mathrm{E} \cdot 2 \\
\end{array}$ & 3 & $\begin{array}{l}3783 \\
3781\end{array}$ & 5.03 \\
\hline & & 108 & & & $\begin{array}{l}3 E .2 \\
-\end{array}$ & 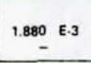 & 3.576 & 7.479 \\
\hline & & 1016 & $\begin{array}{ll}28 & E-1 \\
24 & E-1 \\
\end{array}$ & 3 & $\begin{array}{rl}1.185 & E \cdot 2 \\
1.306 & E \cdot 2 \\
\end{array}$ & & & \\
\hline & & & & 3 & $\begin{array}{rl}9.973 & E \cdot 3 \\
0.082 & E .2 \\
\end{array}$ & 3 & & \\
\hline & & & & & E.3 & & & \\
\hline & & & & & 4 & & & \\
\hline & & $\begin{array}{l}2107 \\
2135\end{array}$ & $\begin{array}{l}3 \in-2 \\
0.2 \\
0.2\end{array}$ & 4 & $\begin{array}{l}2839 \mathrm{E}=3 \\
3014 \\
30.3\end{array}$ & 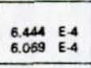 & & .860 \\
\hline & & 2,154 & $6-2$ & 971 & $2.687 \mathrm{E} \cdot 3$ & $3.630 \mathrm{E}-4$ & 1.6 & 4.800 \\
\hline
\end{tabular}

Table 6 Copper sample pair $0.4 \mu \mathrm{m}$ surface finish $448 \mathrm{~N}$ applied force

\begin{tabular}{|c|c|c|c|c|c|c|c|c|}
\hline $\begin{array}{c}\text { TEMERTA } \\
\text { TEATRE } \\
\text { (KELVINI) }\end{array}$ & at & $n$ & $(m w)$ & s, & s, & $1.0_{0}^{\circ}$ & $\begin{array}{l}\text { Tim } \\
\text { ELVVIN) } \\
\end{array}$ & $T_{\max }$ \\
\hline & & $\begin{array}{l}128 \\
121 \\
\end{array}$ & $\begin{array}{l}E-2 \\
E-2 \\
E-2\end{array}$ & $\begin{array}{ccc}1.069 & E-2 \\
1.159 & E-2 \\
\end{array}$ & $\begin{array}{ll}15 & \varepsilon-2 \\
3 & \varepsilon-2 \\
& E-2\end{array}$ & $\begin{array}{l}2.258 \\
2.329 \\
\end{array}$ & & \\
\hline & & 822 & & 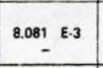 & $\begin{array}{ll}645 \\
- \\
\end{array}$ & & 3.575 & 4.805 \\
\hline & & $\begin{array}{l}2.049 \\
2.030\end{array}$ & $\begin{array}{l}B \\
B \\
1\end{array}$ & 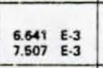 & \begin{tabular}{|l|l|}
1.372 & $E-2$ \\
1.449 & $E .2$ \\
\end{tabular} & & & \\
\hline & 0.311 & 2.053 & E.- & 5330 & 32 E.2 & & & \\
\hline & & $\begin{array}{l}20 \\
20\end{array}$ & & & & & & \\
\hline & & 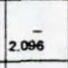 & & 0.434 E:3 & E.3 & & & \\
\hline & & $\begin{array}{l}2.109 \\
2.140 \\
\end{array}$ & $\begin{array}{l}E-2 \\
E .1 \\
\end{array}$ & $\begin{array}{ll}9.211 & \mathrm{E}-4 \\
0.045 & \mathrm{E}-3 \\
\end{array}$ & 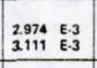 & $5.894 \mathrm{E} \cdot 4$ & 1.3 & $\begin{array}{l}4.899 \\
4.691 \\
4.691\end{array}$ \\
\hline & 2714 & 2.1520 & 7.8286 .2 & $8.962 \mathrm{E}-4$ & $\begin{array}{ll}2.767 & 6.3 \\
2.740 & \mathrm{E} .3 \\
\end{array}$ & $6.365 \mathrm{E}-4$ & 1.689 & ${ }^{4.628}$ \\
\hline
\end{tabular}

Table 7 Copper sample pair $0.4 \mu \mathrm{m}$ surface finish $560 \mathrm{~N}$ applied force

\begin{tabular}{|c|c|c|c|c|c|c|c|c|}
\hline \begin{tabular}{|c|} 
BATM \\
TEMPRATURE \\
(KELVIN)
\end{tabular} & $N / K^{n+1}$ & $n$ & $\alpha_{0}(m w)$ & $\therefore a$ & s. & $8,0_{\circ}^{\circ}$ & $\begin{array}{c}\operatorname{Tmin} \\
\text { (XELVIN) }\end{array}$ & 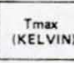 \\
\hline & $\begin{array}{r}0.3992 \\
0.3913 \\
\end{array}$ & $\begin{array}{l}2001 \\
2016 \\
\end{array}$ & $\begin{array}{l}83 \\
03 \\
0.52 .2 \\
\end{array}$ & $\begin{array}{rl}1.273 & E-2 \\
1.320 & E-2 \\
\end{array}$ & $\begin{array}{ll}221 & E-2 \\
E 2-2 & E-2 \\
\end{array}$ & 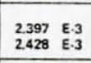 & $3.7 \mathrm{C}$ & $\begin{array}{l}4.858 \\
4.839 \\
4.83\end{array}$ \\
\hline & & 18 & 2 & $=3$ & & & & 739 \\
\hline & & .0.0351 & & & & & & \\
\hline & 0.36 & 2.068 & 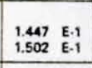 & $\begin{array}{ll}5.998 & E .3 \\
6.602 & E_{-3}\end{array}$ & $\begin{array}{ll}11 & E \cdot 2 \\
6 & 5.2 \\
5 & E-2\end{array}$ & & 3.1. & ${ }_{-532}^{.532}$ \\
\hline & & & 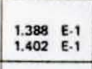 & 3 & & & & - \\
\hline & & & $\begin{array}{ll}54 \\
55-1 \\
E\end{array}$ & $\begin{array}{rl}1.604 & E-3 \\
1.500 & E-3 \\
\end{array}$ & & & & \\
\hline & 0.2749 & - & 2 E.1 & ${ }^{1.054}-E .3$ & $\begin{array}{r}3.096 E .3 \\
-\end{array}$ & $5.807 E-4$ & 1.99 & 4.700 \\
\hline 16 & & 2140 & $E-2$ & 9.700 E-4 & $2.767 \mathrm{E}=3$ & $6.184 \quad E-4$ & 1.689 & 4.528 \\
\hline
\end{tabular}

Table 8 Copper sample pair 0.4 um surface finish $672 \mathrm{~N}$ applied force

\begin{tabular}{|c|c|c|c|c|c|c|c|c|}
\hline $\begin{array}{c}\text { BATH } \\
\text { TEMPERATURE } \\
\text { (KELVIN) }\end{array}$ & $\alpha\left(m w / x^{n+1}\right.$ & $n$ & $a_{0}(m w)$ & s.a & s.n & 1.00 & $\operatorname{Tmin}_{(K \in L V I N)}$ & $\operatorname{Tmax}_{\text {(KELVIN) }}^{\max }$ \\
\hline $\begin{array}{c}3.8 \\
\text { ASCENOING } \\
\end{array}$ & 0.3989 & 2017 & $3.616 \quad E .2$ & 1.3828 .2 & $\begin{array}{lll}2.359 & \varepsilon \cdot 2\end{array}$ & $2.469 \mathrm{E} \cdot 3$ & 3.780 & 4.822 \\
\hline $\begin{array}{c}36 \\
\text { ASCENOING }\end{array}$ & 0.3809 & 2.054 & $8.068 E .2$ & $1.046 \quad E-2$ & $1.918 E .2$ & $2.276 \quad E .3$ & 3.577 & 4.699 \\
\hline $\begin{array}{l}3.4 \\
\text { ASCENOING }\end{array}$ & 0.3928 & 2037 & 1.450 E.1 & 8.623 E.3 & $1.571 \quad E-2$ & $2.169 \mathrm{E} \cdot 3$ & 3.37 & 4591 \\
\hline $\begin{array}{l}3.2 \\
\text { ASCENOING }\end{array}$ & \begin{tabular}{|l|l|} 
\\
\end{tabular} & 2.053 & 1.496 E.1 & $6.674 \quad E-3$ & $1.274 \quad E-2$ & $1.946 \mathrm{E} \cdot 3$ & 3,150 & 4.514 \\
\hline $\begin{array}{l}3.0 \\
\text { ASCENOING }\end{array}$ & 0.3677 & 2.078 & $1.400 \quad \varepsilon .1$ & $5.034 \quad E-3$ & $1.029 E-2$ & $1.685 \quad E .3$ & 2997 & 4.423 \\
\hline $\begin{array}{c}24 \\
\text { ASCENDING }\end{array}$ & 0.3058 & 2118 & 2.200 E.1 & $1.505 \mathrm{E} \cdot 3$ & $3.901 \mathrm{E} \cdot 3$ & $4.936 \mathrm{E}-4$ & 2.378 & 4.640 \\
\hline${ }_{\text {ASCENOING }}^{20}$ & - & - & - & - & - & - & - & - \\
\hline ASCENDING & 0.3013 & 2.151 & $.9 .321 \quad E-2$ & $9.918 \quad E-4$ & $2.794 E=3$ & $6.143 E 4$ & 1.688 & 4.496 \\
\hline
\end{tabular}




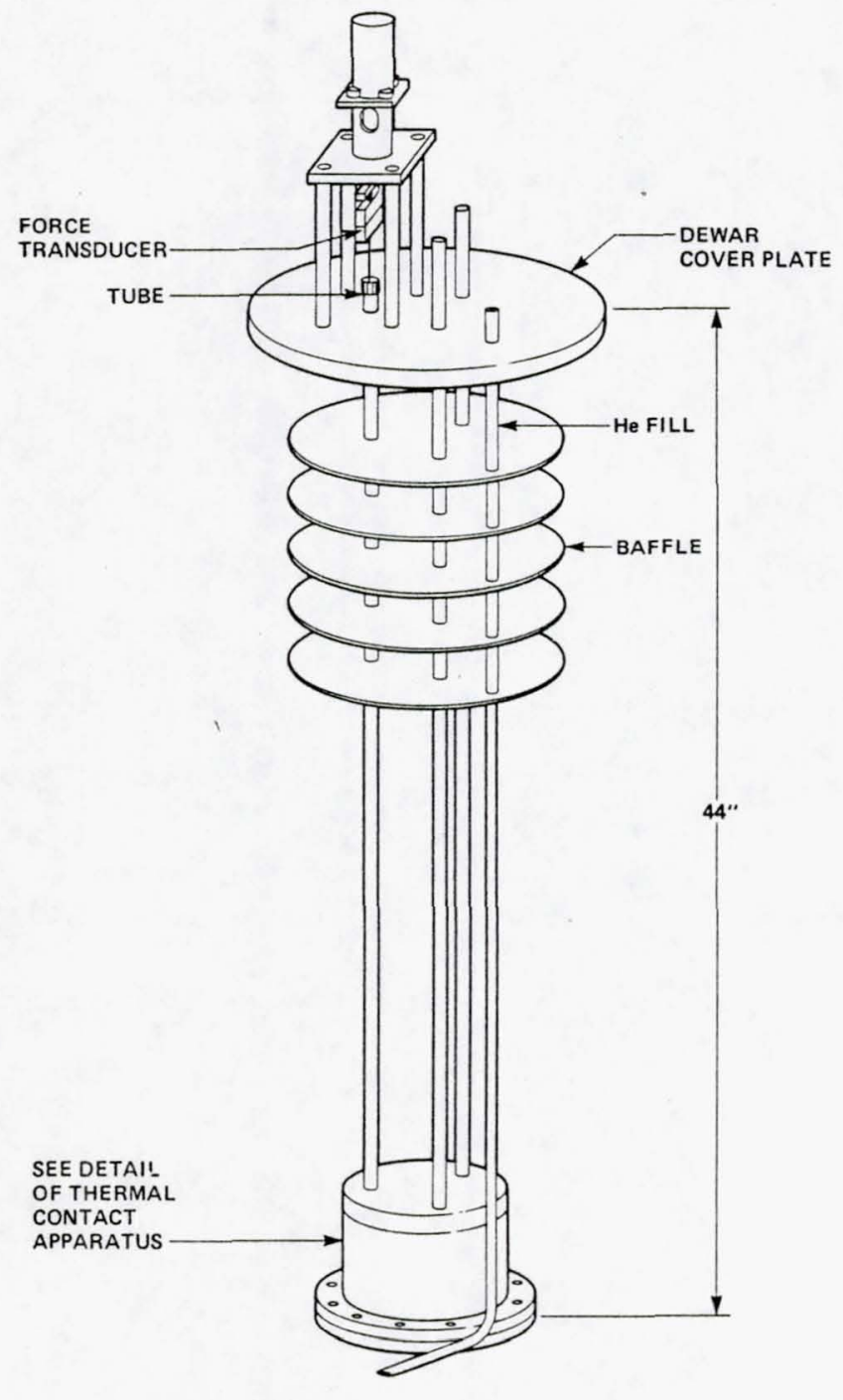

Fig. 1 Overall view of thermal contact apparatus.

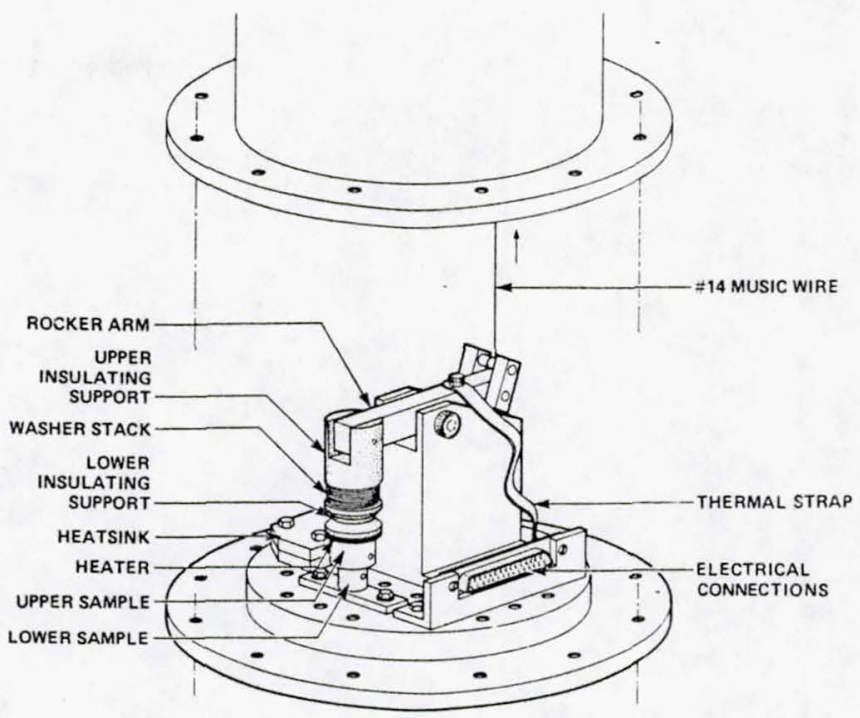

Fig. 2 Detail of cold plate.

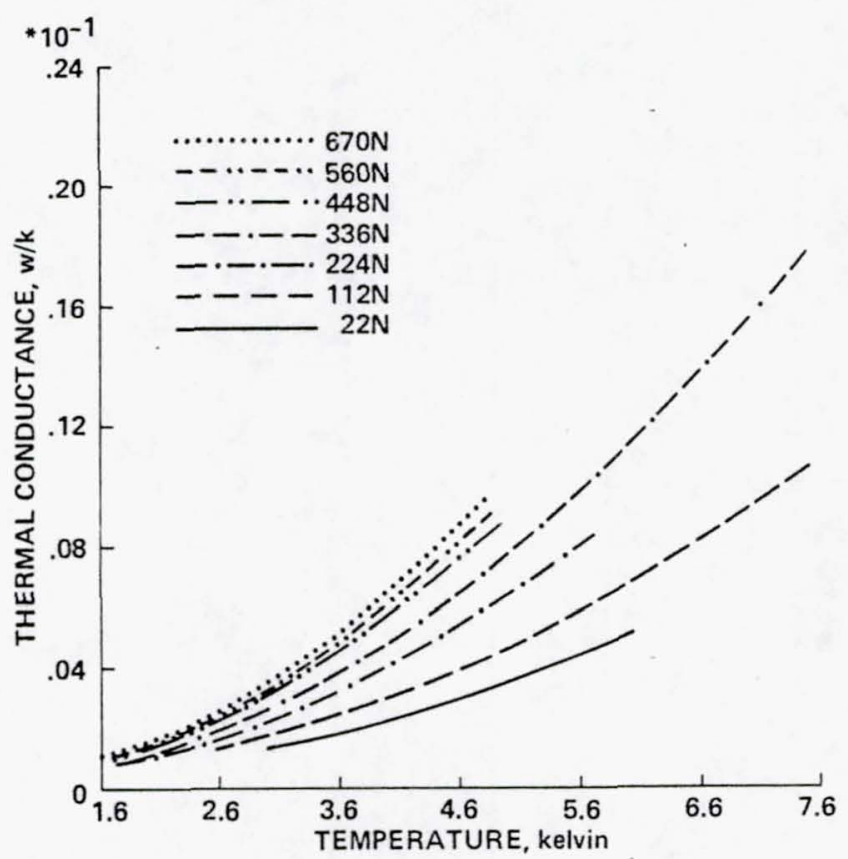

Fig. 3 Results for $0.4 \mu \mathrm{m}$ copper sample pair. 


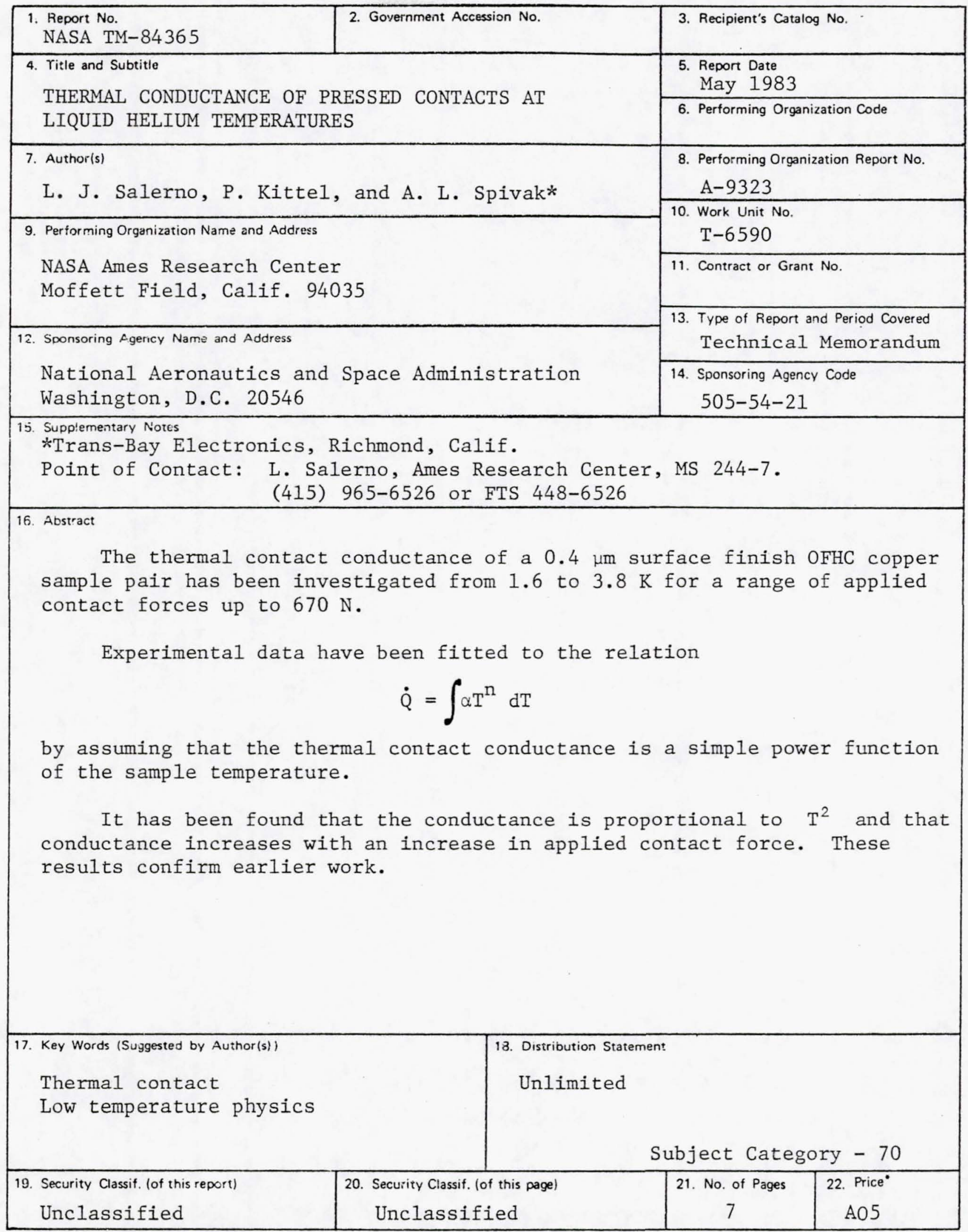

- For sale by the National Technical Information Service, Springfield, Virginia 22161 